\title{
MINERALISED POLYLACTIDE AND POLYCAPROLACTONE SOFT FOAMS WITH HIERARCHICAL MICRO-MACRO POROUS STRUCTURE FOR TISSUE ENGINEERING
}

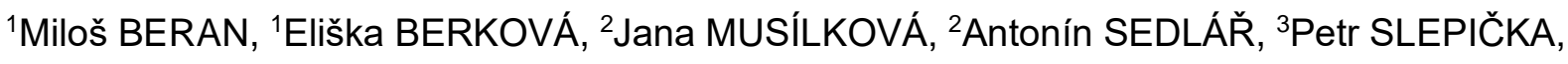 \\ ${ }^{3}$ Dominik FAJSTAVR \\ ${ }^{1}$ Food Research Institute Prague, Prague, Czech Republic, EU, $\underline{\text { m.beran@vupp.cz }}$ \\ 2Institute of Physiology CAS, Prague, Czech Republic, EU, jana.musilkova@fgu.cas.cz \\ ${ }^{3}$ University of Chemistry and Technology, Prague, Czech Republic, EU, petr.slepicka@vscht.cz
}

https://doi.org/10.37904/nanocon.2021.4346

\begin{abstract}
The purpose of the study was development of soft foams from resorbable polymers with unique micro-macro porous interconnected hierarchical structure specially designed as scaffold for engineering of soft tissues.

The foams have been prepared by freeze-drying of solutions of polylactide (PLA) and polycaprolactone (PCL) in 1,4-dioxane. The foams prepared by freeze-drying had interconnected porous aerogel characteristics. The hierarchical structure with bimodal micro-macro pore size distribution were obtained after addition of sucrose or $\mathrm{NaCl}$ porogens with defined crystal size distributions to the solutions before freeze-drying and leaching the porogen crystals from the freeze-dried foams with demineralised water. Polyethyleneimine was chemically conjugated to the alkali-treated foams followed by conjugation of citric acid using carbodiimide chemistry. Finally, they were mineralised by immersing and incubating in a simulated body fluid with ionic concentration similar to that of human blood plasma, to obtain tissue engineering scaffolds. To verify their biocompatibility, the scaffolds were seeded with adipose-derived stem cells (ASC) and sarcoma osteogenic-2 (SaOs-2) human osteoblast-like cells. Morphology of the cells attached to the scaffolds was evaluated and their viability was verified by a metabolic test.

Biocompatibility and usability of the scaffolds was successfully verified by incubation with adipose-derived stem cells and SaOs-2 human osteosarcoma cell line. Mineralised scaffolds are more suitable growth supports for both the cell types than unmineralized collagen scaffolds. The scaffolds have been specially designed for engineering of soft tissues, but they can be used in other categories of tissue engineering, too.
\end{abstract}

Keywords: Tissue engineering, polylactide, polycarolactone, soft foams, hierarchical structure

\section{INTRODUCTION}

Tissue engineering combines isolated functional cells and a biodegradable biomaterial to promote theregeneration of repair diseased or injured tissues. A highly porous scaffold plays a critical role inaccommodating cells and guiding their growth and tissue regeneration in three dimensions. Experiencesand research data have suggested that a pore size between 200 and $300 \mu \mathrm{m}$ might be necessary for the cellseeding efficiency and the homogeneity of the tissue engineered. In the last two decades, a number of foaming techniques have been developed to fabricate highly interconnective, porous scaffolds for tissue engineering applications. Artificial tissue substitute prepared either from nanostructured biocompatible materials or by decellularized non-autologous pericardium combined with autologous ASCs seems to be a promising candidate for cardiovascular grafts capable of accelerating in situ endothelialization. ASCs differentiation could be facilitated by sustained release of growth factors [1]. An advantage of this approach is that ASCs can easily be collected from the patient by liposuction. In this work, we have developed a method of preparation of polymeric foams with hierarchical micro-macro porous structure by freeze-drying of polymeric solutions in 1,4-dioxane with addition of water soluble porogens. 


\section{MATERIALS AND METHODS}

\subsection{Preparation of the soft foams with hierarchical micro-macroporous structure from PLA, PCL and their mixtures}

Polylactide (PLA) Ingeo 4043D, Mn $160.000 \mathrm{~g}^{\mathrm{mol}}{ }^{-1}$ (NatureWorks, USA) and polycaprolectone (PCL), average Mr 80000 (Sigma-Aldrich, Czech Republic) and their different mixtures were used to prepare the soft foams. Single polymers were dissolved in 1,4-dioxane (DO). Concentrations of the polymers, or their mixtures, were in the range $1-5 \%(\mathrm{w} / \mathrm{w})$. Porogens in the form of commercial caster sugar (crystal size in the range $0,16-$ $0,63 \mathrm{~mm}$ ) or finely ground $\mathrm{NaCl}(0,25-0,5 \mathrm{~mm})$ were added to the solutions aligned to the solution surface level. After freezing at $-75^{\circ} \mathrm{C}$ the suspensions were freeze dried in Lyovac GT 2 freeze dryer (LH Leybold, Germany). All chemicals, except PLA, were purchased from Sigma-Aldrich (Czech Republic).

\subsection{Mineralisation of the prepared foams}

The prepared foams were functionalized by mineralization with simulated body fluid (SBF) solution, using carbodiimide chemistry, as described by Jaidev and Kaushik Chatterjee [2]. Composition and preparation of the SBF solution were described by Kobuko and Takadama [3]. All chemicals were purchased from SigmaAldrich (Czech Republic).

\subsection{Microstructure visualization in SEM}

SEM scans were acquired by a scanning electron microscope (FIB-SEM, LYRA3 GMU, Tescan, Brno,Czech Republic). The applied acceleration voltage was $10 \mathrm{kV}$. The examined samples were covered with a $\mathrm{Pt}$ conductive layer of $20 \mathrm{~nm}$ thickness.

\subsection{Sterilization of the prepared foams}

Before the experiments, the samples of foams were sterilized by gamma-irradiation, using the basicsterilization dose (Microtron MT25, Nuclear Physics Institute, Academy of Sciences of the Czech Republic, Rezž).

\subsection{Metabolic aktivity assay}

The metabolic activity of adipose derived stem cells (ASCs) and sarcoma osteogenic-2 (SaOs-2) human osteoblast-like cells (European Collection of Cell Cultures, Cat. No. 89050205) seeded in soft foams scaffolds, incubated in 24 or 96 -well plates was measured with a resazurin assay according to Sedlár, et al. [4]. ASCs were isolated from lipoaspirates and characterized according earlier study [5]. ASCs were incubated in DMEM medium with $10 \%(\mathrm{w} / \mathrm{w}$ ) of fetal serum (FS) and $10 \mathrm{ng} / \mathrm{ml}$ of growth factor FGF 2 . The medium was inoculated with 150 thousand ACS cells in $1,5 \mathrm{ml}$ of the medium per sample. SaOs-2 cells were incubated in McCoys medium with $15 \%$ (w/w) of FS. The medium was inoculated with 200 thousand SaOs-2 cells in $1,5 \mathrm{ml}$ of the medium per sample. The cell metabolic activity was regarded as an indirect marker of the cell proliferation and the cell number. The results of the metabolic activity assay were compared with results obtained with polystyrene (PS) substrate (reference control).

\subsection{Determination of morphology and cell growth}

Morphology and cell growth were determined by a standard method of staining of cytoskeleton with fluorescent and biotinylated derivative of phalloidin. Nuclear DNA was stained with fluorescent4',6-diamidin-2-fenylindol (DAPI). Images obtained by fluorescent microscopy with Olympus IX71 microscope equipped with DP71 digital camera allowed to distinguish cytoskeleton with red colour and cell nuclei with blue colour. Dragonfly confocal microscope (Andor Technology, Great Britain) have been used to visualize ingrowth of the cells into the scaffolds. The results were compared with results obtained with polystyrene (PS) substrate (reference control). 


\section{RESULTS AND DISCUSSION}

The foams prepared by freeze-drying had interconnected microporous aerogel characteristics. This primary pore sizes can be regulated by changing the concentration of the polymers in solution in the range from several $\mu \mathrm{m}$ to several tens of $\mu \mathrm{m}$. The hierarchical structure with bimodal micro-macro pore size distribution were obtained after addition of water soluble porogens with defined crystal size distributions to the solutions before freeze-drying and leaching the crystals from the freeze-dried foams with demineralised water. Photography of the soft foams prepared from PCL/PLA mixture (5:3), with total concentration of polymers in DO solution 4\% $\mathrm{W} / \mathrm{w}$, after leaching the caster sugar porogen is shown in Figure 1A. SEM image of inner structure of the foam is shown in Figure 1B.

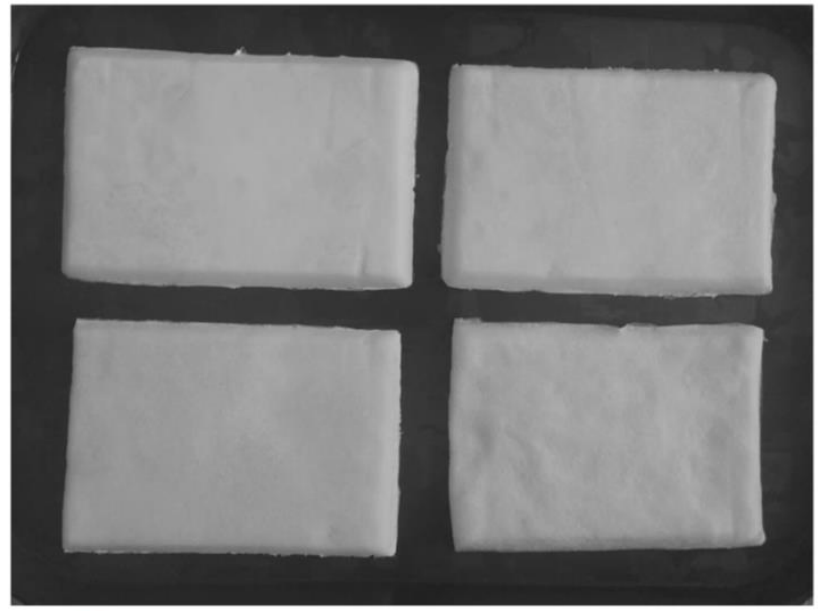

A

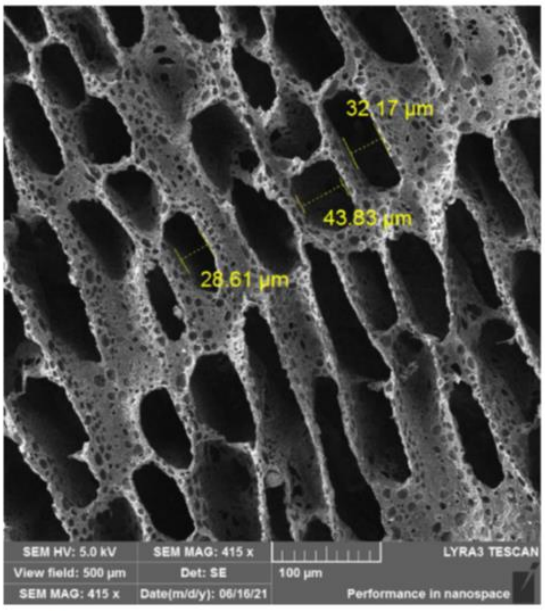

B

Figure $1 \mathrm{~A}$ - photography of preprared PLA/PCL (3:5, total concentration of the polymers in DO $4 \% \mathrm{w} / \mathrm{w})$ foams; $\mathbf{B}$ - SEM image of microstructure of the mineralised scaffold

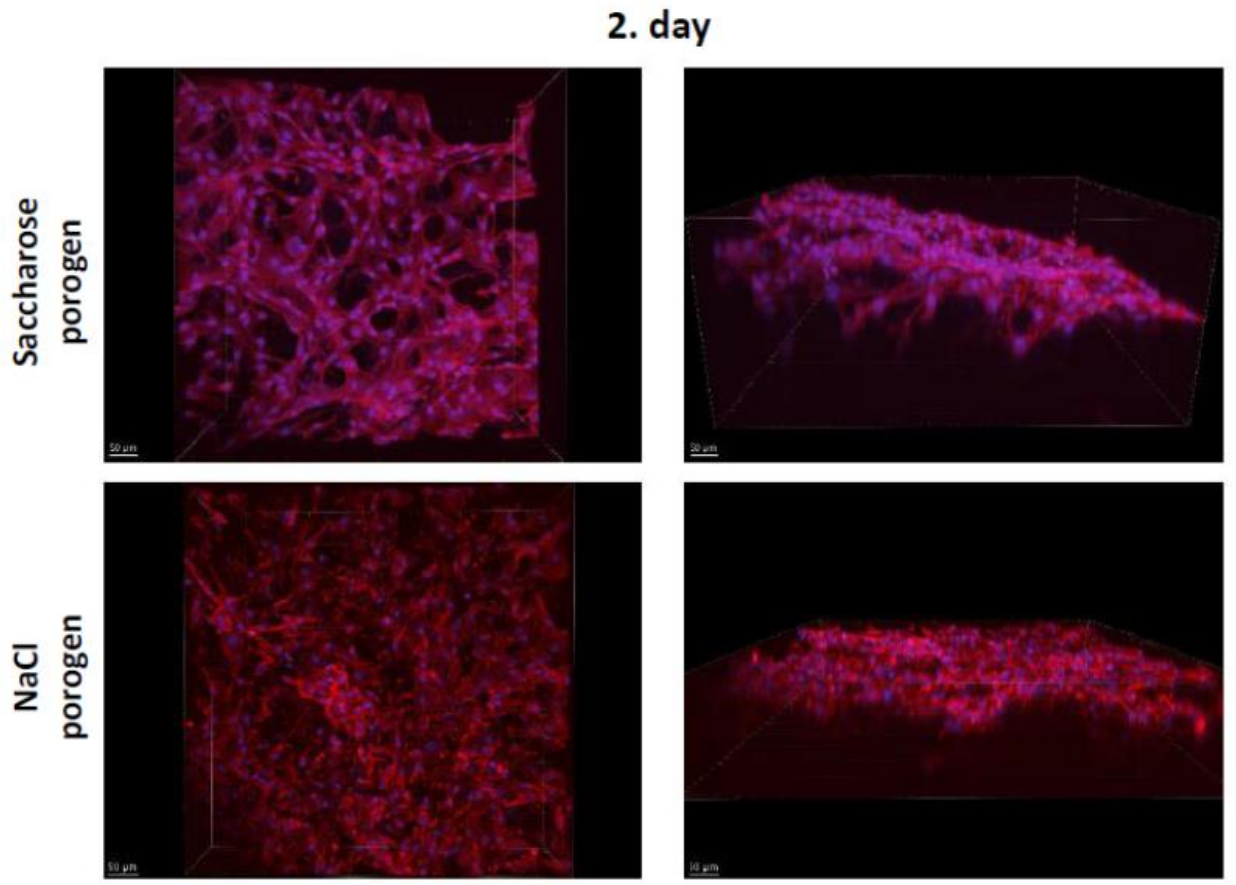

Figure 2 3D photography from the Drangonfly confocal microscope - ACS cells ingrowth and proliferation through the unmineralised PLA/PCL foams 
The inner structures of the foams prepared from PLA and PCL, or their different mixtures, were almost identical at defined concentrations of the polymers. The primary micropores, visible at the image, was formed by dioxane sublimation from the freezed structure. The macropores, formed after the porogen leaching, are slightly smaller than porogen crystal sizes because of partial polymer foam shrinkage after the leaching and drying. The aeras of cross-section of the primary and secondary micropores of the PLA/PCL (3:5) foams prepared with the total concentration of the polymers in DO $4 \% \mathrm{w} / \mathrm{w}$ after leaching the caster sugar porogen were in the range $3,1-314 \mu \mathrm{m}^{2}$ and $2400-7800 \mu \mathrm{m}^{2}$, respectively. Figure 2 shows visualisation of ingrowth and proliferation of ASCs into the unmineralised scaffolds. The cells are mainly localised at the surface of the scaffold with a limited ingrowth into the foam interior. We suppose, that the main reason of the limited ingrowth of the cells is hydrophobicity of the surface resulting in restricted suction of aqueous media.

The metabolic activity of ASC and SaOs-2 cells cultivated on different PLA/PCL foam types was tested. For the unmineralised foams the ASCs as a tested cells were chosen. For the testing of the mineralisation effectiveness of both the stem cells (ASCs) and the osteoblast-like cells (SaOs-2) were used. The metabolic activity of ACS cells cultivated on unmineralised PLA/PCL foams in comparison with polystyrene (PS) as a positive control taken on 10. day after seeding are shown at Figure 3A. We found out, that the metabolic activity of the cells cultivated on tested scaffolds was considerably high, around $40 \%$ of the value of the positive control (PS). Figure 3B shows the comparison of metabolic activity the both ASCs and SaOs-2 cells cultivated on unmineralised (C) and mineralized (M) PLA/PCL foams on 2. day (blue) and 6. day (orange) in comparison with polystyrene (PS) as a positive control. The ASCs and SaOS-2 cells metabolic activities after 6 days of cultivation on the mineralised scaffolds were comparable despite the fact, that SaOS-2 grow usually slowly than ASCs cells, as was shown on PS.

The cells growing on the PLA/PCL foams were visualized by fluorescence staining. Figure 4 shows results of fluorescent staining of SaOs-2 cells (blue colour) incubated in unmineralised and mineralised scaffolds prepared with the saccharose porogen and Figure 5 results of fluorescent staining of ASCs (blue colour) incubated on mineralised scaffolds prepared with the saccharose porogen in comparison with PS substrate control. Both the cell types formed rather single layer on the scaffold surface on day 2 . In the following days the cells formed more layers and started to penetrate into the scaffold interior, as noticeable from higher density of the cell nuclei and morphology of the cells at higher magnification. More distinct fibrillar structure (red colour) testify in favour of ASCs differentiation towards smooth muscle. The metabolic activities of both the cell types cultivated on the both mineralized and unmineralized PLA/PCL foams are rising during the time interval between the day 2 and day 6 . The metabolic activities of both the cell types were getting closer to the metabolic activities on the reference PS substrate surface, despite the fact, that a part of the cells is hidden inside 3D structure of the foams. That means, that PLA/PCL foams of both types are biocompatible and appropriate for the cell cultivation.
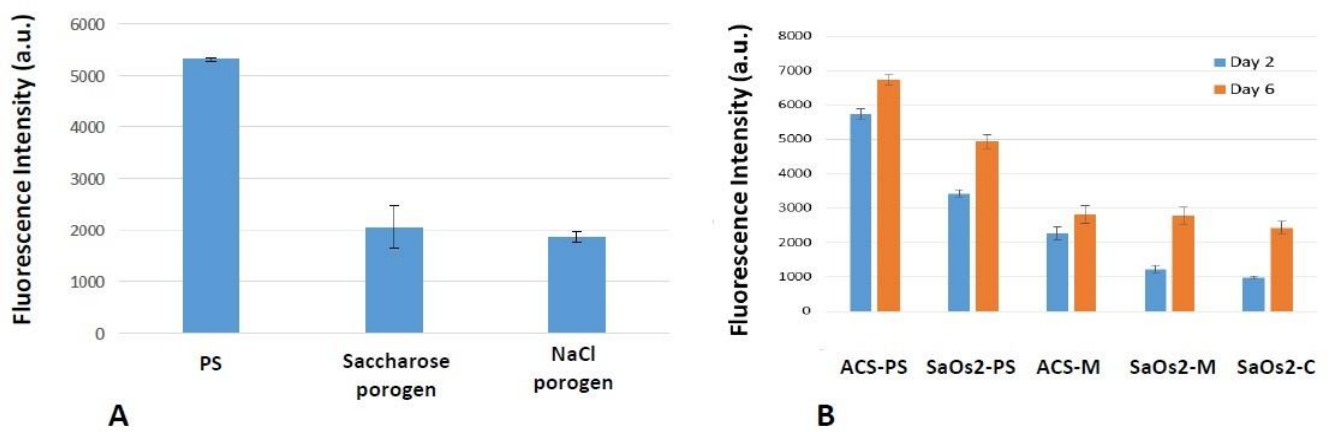

Figure $3 \mathbf{A}$ - metabolic activity of ASCs cultivated on unmineralised PLA/PCL foams prepared with saccharose $\mathrm{NaCl}$ porogens in comparison with ASCs cultivated on polystyrene (PS) support on day 10. after cell seeding; B - metabolic activity of ASCs or SaOS-2 cells cultivated on mineralised (M) and unmineralised (C) PLA/PCL foams prepared with saccharose porogen with in comparison with polystyrene (PS) support.

Samples tested on 2. day (blue) and 6. day (orange) after cell seeding. 
Day 2

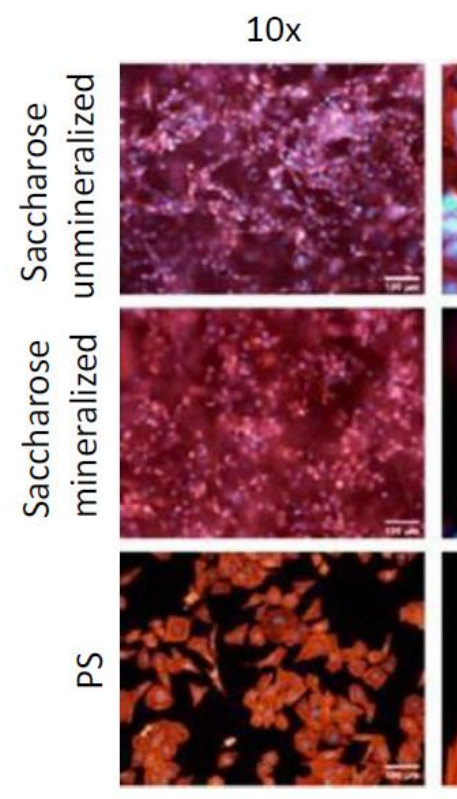

$40 x$
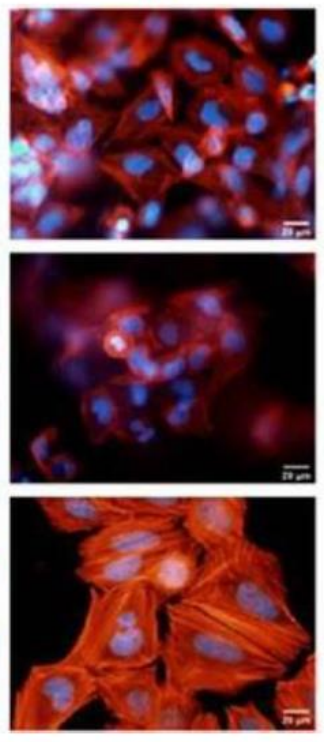

Day 6
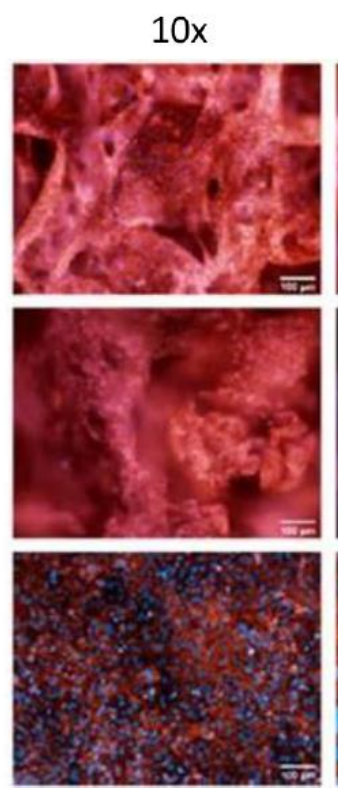

$40 x$
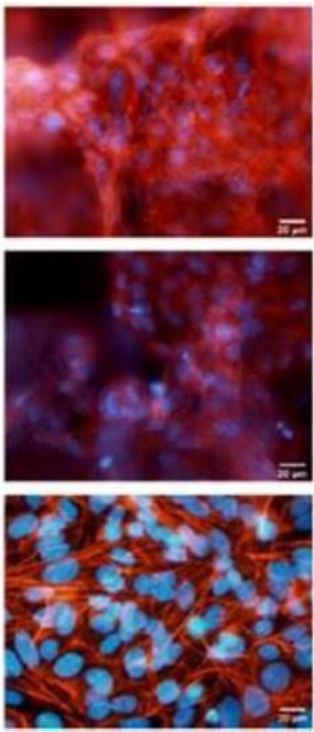

Figure 4 Fluorescent staining of SaOs-2 cells (blue colour) incubated in native and mineralised scaffolds prepared with saccharose porogen and PS control substrate

Day 2
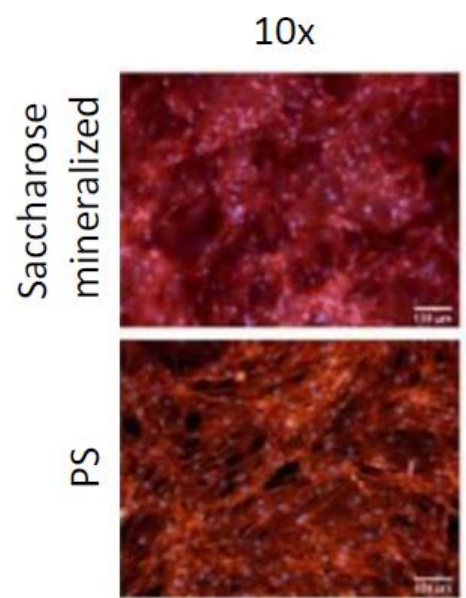
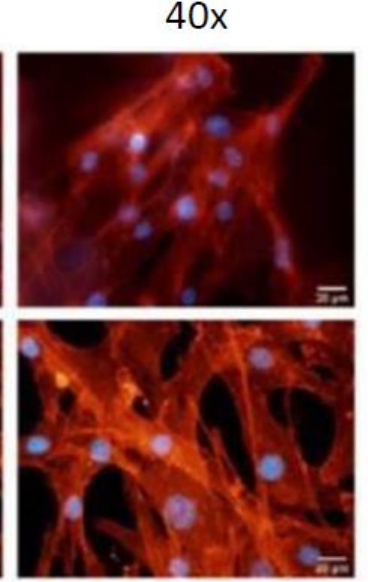

Day 6

$10 x$
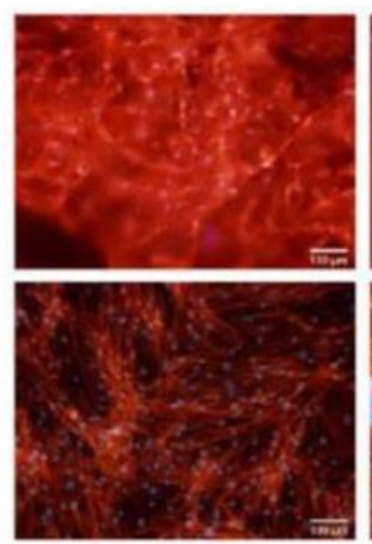

$40 x$

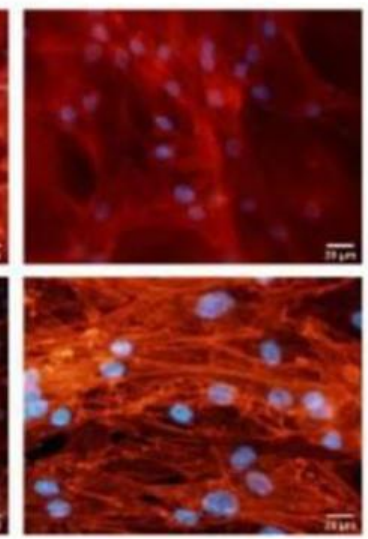

Figure 5 Fluorescent staining of ACS cells (blue colour) incubated in mineralised scaffolds prepared with saccharose porogen and PS control substrate

Polymeric foams find uses in variety of applications including packaging, thermal and acoustic insulation, energy dissipation, shock protection, filtration and separation. Hierarchically structured porous materials have attracted much attention because of their diversity and good performance, and are largely used as an important family of functional materials in catalysis, photocatalysis, adsorption, separation, energy conversion and storage, sensing, and biomedicine in recent years [6]. We suppose, that low density and well controlled and interconnected hierarchical porosity at different length scales will facilitate in-vivo resorption of the foam scaffolds for tissue engineering and improve nutrients diffusion into and metabolites diffusion outside the foam scaffolds. DU, Y., et al. [7] already described preparation of PCL nanofibrous scaffolds with hierarchical pore structure for tissue engineering. Macro/microporous poly(lactic-co-glycolic acid)/polyethylene glycol composite 
scaffold architectures have been developed using a hybrid solid freeform fabrication/thermally induced phase separation technique [8]. PCL foams with a well-defined micrometric and bimodal open-pore dimension distribution, suitable as scaffolds for tissue engineering, were prepared by SALERNO, A., et al. [9]. They prepared porous network pathway by combining gas foaming and selective polymer extraction techniques with thermoplastic gelatin. Dual porosity protein-based scaffolds with enhanced cell infiltration and proliferation were prepared by other authors [10] using the combination of foaming and freeze-drying. In our study, we have described simple and fast alternative method to prepare polymeric foams with hierarchical micro-macro porous structure suitable for tissue engineering.

\section{CONCLUSIONS}

Soft foams with micro-macro porous hierarchical structure have been prepared by freeze-drying of solutions of PLA and PCL mixtures in 1,4-dioxane with or without addition of sucrose or sodium chloride porogens. The foams prepared by freeze drying had interconnected porous aerogel characteristics. The hierarchical structure with bimodal micro-macro pore size distribution were obtained after addition of the porogens with defined crystal size distributions to the solutions before freeze drying and leaching the porogen crystals from the freeze-dried foams with demineralised water. The freeze dried foams were mineralised using carbodiimide chemistry. Biocompatibility and usability of the scaffolds was successfully verified by incubation with adiposederived stem cells and SaOs-2 human osteosarcoma cell line. Mineralised scaffolds are more suitable growth supports for both the cell types than unmineralized collagen scaffolds. The scaffolds have been specially designed for engineering of soft tissues, but they can be used in other categories of tissue engineering, too.

\section{ACKNOWLEDGEMENTS}

\section{This work was supported by the Czech health research council project NU20-08-00208 and by the Institutional Development Fund of FRIP provided by the Ministry of Agriculture of the Czech Republic.}

\section{REFERENCES}

[1] FILOVÁ, E., et al. Accelerated in vitro recellularization of decellularized porcine pericardium for cardiovascular grafts. Biomedical Materials. 2021, vol. 16, 025024.

[2] JAIDEV, L.R., KAUSHIK CHATTERJEE. Surface functionalization of 3D printed polymer scaffolds to augment stem cell response. Materials \& Design. 2019, vol. 61, pp. 44-54.

[3] KOKUBO, T., TAKADAMA, H. How useful is SBD in predicting in vivo bone aktivity? Biomaterials. 2006, vol. 27, pp. 2907-2915.

[4] SEDLÁŘ, A., et al. Growth Factors VEGF-A165 and FGF-2 as Multifunctional Biomolecules Governing Cell Adhesion and Proliferation. Int. J. Mol. Sci. 2021, vol. 22, p. 1843.

[5] TRÁVNÍČKOVÁ, M., et al. Differentiation of adipose tissue-derived stem cells towards vascular smooth cells on modified poly(L-lactide) foils. Biomedical Materials. 2021, vol. 16, 025016.

[6] LIANG, W., ZHENGYI, F., BAO-LIAN, S. Hierarchically structured porous materials: synthesis strategies and applacations in energy storage. National Science Review. 2020, vol. 7, pp. 1661-1701.

[7] DU, Y., CHEN, X., LEI, B. Facilely Fabricating PCL nanofinrous scaffolds with hierarchical pore structure fot tissue engineering. Materials Letters. 2014, vol. 14, pp. 62-65.

[8] AKBARZADEH, R., et al. Hierarchical polymeric scaffolds support the growth of MC3T3-E1 cells. J. Mater. Sci: Mater. Med. 2015, vol. 26, pp. 116.

[9] SALERNO, A., et al. Design and Preparation of $\mu$-Bimodal Porous Scaffold for Tissue Engineering. Journal of Applied Polymeric Science. 2007, vol. 106, pp. 3335-3342.

[10] RASOULIANBOROUJENI, M., et al. Dual Porosity Protein-based Scaffolds with Enhanced Cell Infiltration and Proliferation. Sci Rep. 2018, vol. 8, 14889. 